\title{
Decision-making for unmanned aerial vehicle operation in icing conditions
}

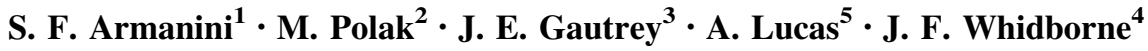

Received: 29 January 2016/Revised: 22 September 2016/ Accepted: 22 September 2016/Published online: 1 October 2016

(c) The Author(s) 2016. This article is published with open access at Springerlink.com

\begin{abstract}
With the increased use of unmanned aerial systems (UAS) for civil and commercial applications, there is a strong demand for new regulations and technology that will eventually permit for the integration of UAS in unsegregated airspace. This requires new technology to ensure sufficient safety and a smooth integration process. The absence of a pilot on board a vehicle introduces new problems that do not arise in manned flight. One challenging and safety-critical issue is flight in known icing conditions. Whereas in manned flight, dealing with icing is left to the pilot and his appraisal of the situation at hand; in unmanned flight, this is no longer an option and new solutions are required. To address this, an icing-related decision-making system (IRDMS) is proposed. The system quantifies in-flight icing based on changes in aircraft performance and measurements of environmental properties, and evaluates what the effects on the aircraft are. Based on this, it determines whether the aircraft can proceed, and whether and which available icing protection systems
\end{abstract}

S. F. Armanini

s.f.armanini@tudelft.nl

1 Faculty of Aerospace Engineering, Section Control and Simulation, Delft University of Technology, Kluyverweg 1, 2629HS Delft, The Netherlands

2 Institute of Flight System Dynamics, Technische Universität München, Boltzmannstraße 15, 85748 Garching, Germany

3 National Flying Laboratory Centre, School of Aerospace, Transport and Manufacturing, Cranfield University, Bedfordshire MK43 0AL, UK

4 Dynamics, Simulation and Control Group, School of Aerospace, Transport and Manufacturing, Cranfield University, Bedfordshire MK43 OAL, UK

5 AOS Group Ltd, Sheraton House, Castle Park Cambridge, CB3 0AX, UK should be activated. In this way, advice on an appropriate response is given to the operator on the ground, to ensure safe continuation of the flight and avoid possible accidents.

Keywords Aircraft icing · Ice detection · Unmanned aerial system $\cdot$ Decision-making

\section{Introduction}

The widespread interest in a more extensive use of unmanned aerial systems (UAS) in the civil sector has led to significant efforts towards the integration of UAS in unsegregated airspace [1]. This involves improving the safety and reliability of UAS and developing new regulations specifically for such systems. One of the challenges in this process, is the regulation and certification of UAS flight in icing conditions. Some UAS, e.g., the MQ-4C Triton, are currently equipped with ice protection systems, but this capability does not extend to continuous operations in icing.

In-flight icing degrades aircraft performance and control, is a safety hazard to manned and unmanned aircraft alike and is still a cause of accidents [2, 8]. Extensive research has been conducted to study the effects of icing through flight tests, wind tunnel tests and simulations $[4,5,11,13,19,21,25,28,33]$. It was shown that ice accretion increases drag significantly even in short exposure times $[4,25,33]$, affecting especially the lift-independent component [33]. Icing was also found to reduce the maximum lift coefficient, lift curve gradient and stall angle of attack [10]. The reduction in tail lift, in particular, results in reduced stability $[28,33]$, while flow separation behind ice shapes changes the control surface hinge moments reducing control effectiveness [28]. 
Aircraft likely to encounter icing conditions in their routine operations are equipped with ice protection systems (IPS), which reduce but do not eliminate the effects of icing [25] and thus can allow for flight in icing conditions up to a certain severity. Currently, however, the activation and operation of the IPS relies largely on the pilot's judgement and view of the airframe. Similarly it is often left to the pilot to decide how to handle each situation and determine whether safe operation is still possible or whether it is necessary to fly out of icing conditions, based on aircraft-specific procedures and on the experience or intuition-guided interpretation of aircraft behaviour and sensory cues. While this approach is often adequate, the enduring hazard of icing has led to research aimed at assisting pilots in such situations. This work mainly focuses on in-flight characterisation of icing, to provide better information to the pilot, and either warn them of the reduced flight envelope or directly account for this in the flight control system. While focused on manned aircraft, this work provides a useful basis for the consideration of UAS, where the problem of icing is enhanced due to the absence of an on-board pilot.

At present, there is no uniform method to describe inflight icing and definitions used in aviation are mostly qualitative [20, 32], partly because the effects of icing are aircraft dependent. One approach suggested to characterise icing in flight is to use some form of parameter identification to estimate aircraft stability and control derivatives in flight, and compare these to the corresponding uniced values or to pre-computed icing affected values $[9,14,16-18,23,29]$. A drawback is the typically high computational burden of online parameter estimation. This is particularly relevant when considering application on UAS, which are often small and have a limited payload and computational power availability. It is important to use only measurements that are reliable and can be easily obtained in flight, and to minimise computational requirements. A further limitation of online parameter estimation is the requirement for dynamic excitation, which is not available in steady-state flight unless actively introduced. In steady flight, a possible alternative is to identify changes in trim conditions caused by icing [11]. This approach has been judged applicable, based on experimental results, but has not been translated into a practical detection mechanism.

A small number of studies have also proceeded beyond icing characterisation, suggesting new approaches to ice protection that rely less on human judgement and could support pilots in handling critical situations. Bragg et al. $[9,12]$, in particular, proposed a smart icing system that uses parameter identification to detect icing in flight, and based on this information takes appropriate measures, such as activating the IPS and modifying the flight envelope for the flight control system. A similar approach could be used for UAS; however, the absence of a pilot, even in a purely supervisory role, must be considered.

Icing can be complex to characterise using measurements only. While dedicated icing sensors are emerging that might provide reliable direct measurements, in general it remains essential for the pilot to assess each specific situation and determine an appropriate response to it. For UAS, this solution is not applicable; therefore, the problem of icing is particularly critical. Currently, UAS are not able to operate in known icing conditions and this can be a significant limitation, e.g. in search and rescue operations. Hence the need was identified for a system that emulates the reasoning process of a pilot in this context, i.e. that detects and quantifies icing, and assesses the overall situation and available data to reach an informed decision in response to the perceived situation.

Existing work has considered the problem of icing detection, in limited cases also for small unmanned aircraft $[16,30,31]$, and, to a lesser extent, has considered the possibility of offering advice to the pilot on how to handle icing. However, unmanned flight in icing with autonomous handling of such situations has not been widely studied yet.

This paper presents the development of an icing-related decision-making system (IRDMS) that aims to assist safe unmanned flight in icing conditions and perform the main tasks typically performed by a pilot. The requirements are for the system to (1) detect the formation of ice and quantify it, and (2) determine an appropriate response to the perceived conditions. Two aspects are addressed: (1) possible means for the detection and quantification of icing in flight, and (2) the appropriate evaluation of and response to icing-related situations in the absence of an on-board pilot. The proposed system would have an advisory role and assist the operator on the ground in responding appropriately to critical weather conditions and help to ensure continued safe operation without requiring the UAS to wholly avoid potential icing conditions, which currently poses a significant restriction on their application. Such a system will be an important step in enhancing the safety and all-weather capability of UAS and in aiding the process of their integration in unsegregated airspace.

This paper is structured as follows. Section 2 describes the two suggested icing detection and quantification methods, which infer icing from changes in drag and in trim settings, respectively. Section 3 introduces the envisaged IRDMS and outlines the decision-making process, including the evaluation of the icing situation from measurements and the determination of appropriate responses to it. Section 4 discusses initial simulation and flight tests used to assess the detection approaches and to test the functionalities of the IRDMS. Section 5 summarises the main conclusions and provides suggestions for further work. 


\section{Icing characterisation in flight}

The first part of this work concerns the investigation of methods to detect and quantify icing in flight in the absence of an on-board pilot. Two approaches were evaluated, one based on changes in drag, the other on changes in trim. In both cases, the underlying idea is to continually compare clean and icing-affected values of specific variables known to be significantly influenced by icing and relevant to aircraft performance. Differences between the two values are used to detect and to some extent quantify icing.

In aviation forecasts, icing is typically described using the following designations: none, trace, light, moderate, severe [32]. It has been argued that these are mostly qualitative and can be difficult to apply [20], but they remain a viable solution thanks to the on-board pilot. Using a combination of sensory cues and experience, the pilot can generally make a reasoned appraisal of the situation and react accordingly. For UAS, however, qualitative definitions are insufficient, thus our aim was to define quantitative icing intensity scales.

Since icing is an aircraft-dependent process, the Cranfield University National Flying Laboratory Jetstream J31 was chosen as a test platform to investigate the proposed detection methods. The use of an instrumented manned aircraft for initial investigations is more convenient and it allows for pilots to assess the performance of the suggested system and compare its evaluations to their own. Evidently, the use of a specific test platform also implies that the evaluations made apply more closely to vehicles of similar size and weight. Similarly, it is important to note that the detection approaches considered constitute a baseline, and their suitability for a particular vehicle must be evaluated on a case-by-case basis prior to any real-world application. The methods would have to be extended and adapted in order to be applied to a specific vehicle.

At this stage, the focus is general and practical implementation issues are not addressed in significant detail. However, it is essential to bear these points in mind from the outset. It must for instance be considered that, while the suggested approaches are based on standard measurements, some of these measurements must be highly accurate and adequate instrumentation may not be available on very small UAS. It can be argued that if a vehicle is intended to operate routinely in potentially hazardous conditions, it will be equipped accordingly. Alternatively, the quality of measurements may also be improved by means of filtering or sensor fusion. Measurements may also be influenced by other factors unrelated to icing, e.g. turbulence or wind, which may complicate the detection process. Especially very small aircraft would be highly affected. One way of mitigating this is to consider several detection methods simultaneously (cf. Sect. 3.1), which in many cases may allow for an adequate assessment to be made, even if single measurements are not unequivocal. Finally, controllers running on-board would affect the proposed detection approaches and would have to be taken into account in the implementation.

The focus of this study is steady level flight, which is the most common flight regime for most aircraft and where excitation is generally insufficient for effective parameter estimation. This is considered a logical starting point to study the problem and allow for initial development of the IRDMS, before proceeding to more complex detection methods that consider manoeuvring flight. The chosen icing characterisation approaches have a limited applicability due to the assumptions they are based on. However, if found to be sufficiently effective, these detection approaches would be highly advantageous for implementation on small UAS with limited payload and computational power, in view of their considerable simplicity. The proposed methods are outlined in the following sections. Further detail can be found in $[6,24]$.

\subsection{Drag-based icing detection}

The first icing detection approach is based on drag changes detected in flight. Drag increase is one of the most immediate and significant consequences of ice accretion. While stability and control degradation can be considered more detrimental and likely to lead to accidents, these typically occur at a later stage. Given that the aim of the proposed system is to detect icing early on and avoid situations where aircraft stability and control are significantly affected, drag was considered an appropriate icing indicator. Further to this, drag changes manifest themselves clearly as changes in aircraft performance, which are straightforward to determine from standard on-board measurements available even on small UAS.

To detect icing, a continual comparison is made between the on-board determined drag coefficient, potentially affected by icing, and the drag coefficient for the uniced aircraft in the same trimmed flight conditions. Clean values for the drag coefficient $C_{\mathrm{D}}$ can be obtained from the wellknown equation,

$$
C_{\mathrm{D}, \text { clean }}=C_{\mathrm{D} 0 \text {,clean }}+k C_{\mathrm{L}, \text { clean }}^{2}
$$

where the constant $k$ and the zero-lift drag $C_{\mathrm{D} 0}$ are typically known for a given aircraft, and under the assumption of steady level flight made here, the lift coefficient $C_{\mathrm{L}}$ is given by,

$$
C_{\mathrm{L}, \text { ideal }}=\frac{2 m g}{\rho_{0} S V_{\mathrm{E}}^{2}}
$$


where $V_{\mathrm{E}}$ is equivalent airspeed, $\rho_{0}$ is air density at mean sea level and $S$ is the reference area. Thus the clean aircraft drag coefficient, needed as a reference value, can be obtained in steady flight if the constants in Eqs. 1 and 2 are known and aircraft mass and velocity can be computed online. For clarification it should be noted that the clean lift coefficient can be determined from Eq. 2 even when the aircraft is affected by icing. Assuming the desired velocity is maintained by increasing thrust and the aircraft remains in the same steady level flight condition, the total lift force generated must be the same regardless of icing. While the lift coefficient is affected by icing, the lift force must be kept constant to maintain level flight, e.g. by increasing the angle of attack. Further, it should be noted that while the lift coefficient is a function of the angle of attack, this dependence does not affect the calculation of the ideal lift coefficient from the force required to support the weight, and hence does not need to be specified at this stage.

An estimate of the drag effectively experienced in flight can be obtained, under the assumption of steady level flight and small angles, from the following equation,

$$
C_{\mathrm{D}, \text { actual }}=\frac{2 T}{\rho_{0} S V_{\mathrm{E}}^{2}}
$$

where $T$ is the total thrust produced by the engines. Maintaining the same velocity in the presence of higher drag requires higher thrust and thus implies a larger drag coefficient.

Differences between the results given by Eqs. 1 and 3 can be used to detect ice forming on the airframe. The next step is to derive a quantitative scale for icing and its effects. For this study, empirical data for the Jetstream 31 were used to derive an approximate model relating changes in performance to icing-related changes in drag, and to define icing severity levels corresponding to these changes. Specifically, the Jetstream Crew Manual “Advisory Performance Information for Flight with Accreted Ice" [7] contains information on how icing affects the aircraft's enroute climb performance. This allowed for a measure of the effects of icing on drag to be obtained for the level of icing considered in the manual, which was assumed to be moderate, the maximum level permitted for this aircraft.

The climb gradient is defined as,

$\operatorname{grad}=\frac{\mathrm{d} h}{\mathrm{~d} x}=\frac{\mathrm{d} h}{\mathrm{~d} t} \frac{1}{V}$

where $h$ is altitude, $x$ is distance, $\frac{\mathrm{d} h}{\mathrm{~d} t}$ is rate of climb, and $V$ is velocity (airspeed). When icing is known or suspected on any part of the aircraft, the flight manual prescribes a specific increase in velocity for en-route climb. It follows from Eq. 4 that if a given climb rate is to be maintained, a higher velocity must lead to a lower climb gradient, regardless of the presence of ice on the airframe. Additionally, the rate of climb for a given velocity is lower with accreted ice due to the increased drag and consequent reduction in available excess power. The manual gives the decrease in climb gradient associated with the increase in velocity alone, as well as the total climb gradient reduction with ice on the airframe. From these values, the climb gradient reduction associated with the presence of ice alone, $\Delta \operatorname{grad}_{\text {ice }}$, can be determined. The change in rate of climb due to icing, at the considered velocity, can then be obtained from Eq. 4, and this change can be related to the corresponding change in available excess power,

$\frac{\mathrm{d} h}{\mathrm{~d} t}=\frac{P_{\mathrm{a}}-P_{\mathrm{r}}}{\mathrm{mg}}-\frac{V}{g} \frac{\mathrm{d} V}{\mathrm{~d} t}$,

where $P_{\mathrm{a}}$ is available power, $P_{\mathrm{r}}$ is power required to maintain the specified flight condition, $m$ is mass, $g$ is acceleration due to gravity, and $V$ is velocity (true airspeed). Under the assumption of a constant velocity this simplifies to

$\frac{\mathrm{d} h}{\mathrm{~d} t}=\frac{P_{\mathrm{a}}-P_{\mathrm{r}}}{\mathrm{mg}}$

Eq. 6 yields an expression for the reduction in available excess power due to icing in terms of the change in attainable rate of climb, or equally in terms of climb gradient and velocity.

$\Delta\left(P_{\mathrm{a}}-P_{\mathrm{r}}\right)_{\text {ice }}=\Delta P_{\text {excess,ice }}=\operatorname{mg}\left(\Delta \frac{\mathrm{d} h}{\mathrm{~d} t}\right)=\operatorname{mg} V\left(\Delta \operatorname{grad}_{\text {ice }}\right)$

The increase in drag due to icing can now be obtained from the reduction in power availability,

$\Delta D_{\text {ice }}=\frac{\Delta P_{\text {excess }, \text { ice }}}{V}=\frac{\operatorname{mg} V\left(\Delta \operatorname{grad}_{\text {ice }}\right)}{V}=\operatorname{mg} \Delta \operatorname{grad}_{\text {ice }}$

and the corresponding change in drag coefficient is given by

$\Delta \mathrm{C}_{\mathrm{D}, \text { ice }}=\frac{2 \Delta \mathrm{D}}{\rho \mathrm{V}^{2} \mathrm{~S}}$

The data in the flight manual describe the reduction in climb gradient at a specific velocity. To apply the information obtained at different flight conditions, in this case velocities, a velocity-independent quantity is needed. As icing has a significant effect on the zero-lift drag (cf. Sect. 1), it was assumed in this context that the icing-induced drag increase is entirely lift independent. The zerolift drag coefficient is approximately independent of velocity at the low subsonic velocities in the operating range of the Jetstream. Under these assumptions, the $\Delta C_{D, i c e}$ value computed from Eq. 9 wholly flows into the 
lift-independent drag component and is independent from velocity.

The data only yield a single drag coefficient decrease, for a particular icing severity. The final step consists in deriving a scale from this value. The obtained threshold values for different icing severity levels, expressed in terms of the drag coefficient, are reported in Table 1 . They were derived under the assumption that the aircraft manual refers to moderate icing, and that drag increases linearly with increasing ice accretion. The latter may not be the case, particularly when larger accretion builds up, however it is considered an acceptable first approximation. Initial flight tests (cf. Sect. 4.2) were conducted to investigate the accuracy of the assumptions made, and further testing is planned to improve the current values and develop a more accurate scale.

\subsection{Trim-based detection}

As an alternative to the drag-based detection approach, changes in trim were explored as a possibility to characterise ice accretion on the aircraft. For this, a relationship was developed between icing and the changes in elevator trim required to maintain a particular steady flight condition [24]. These can be expressed in terms of either the angle of attack or the elevator deflection.

In steady level flight, the elevator is used to maintain the necessary angle of attack at which lift counterbalances weight, and the throttle is used to counterbalance drag with thrust and maintain a constant velocity. In icing, the angle of attack must increase for the same constant velocity and altitude to be maintained, due to the diminished wing lift curve slope and potentially increased weight due to ice build-up. Hence the elevator must be deflected upwards. Additionally, given that the tailplane lift is similarly degraded by icing, elevator effectiveness is decreased. This means that larger deflections are needed to achieve the same effect, thus the necessary change in deflection to maintain trimmed conditions is further increased. At the same time, note that maintaining a constant velocity, as required for this method, also entails increasing thrust because the icing increases the drag. Moreover, the larger angle of attack needed to maintain the original velocity

Table 1 Icing severity levels in terms of change in zero-lift drag coefficient, derived from empirical data for the Jetstream 31

\begin{tabular}{ll}
\hline$\left|\Delta C_{\mathrm{D} 0}\right|$ & Icing severity \\
\hline$<0.005$ & None \\
$0.005-0.015$ & Trace \\
$0.015-0.030$ & Light \\
$0.030-0.045$ & Moderate \\
$>0.045$ & Severe \\
\hline
\end{tabular}

despite the reduced lift also leads to increased drag and hence a further increase in required thrust.

Applying this method requires clean reference values and in-flight determined ones for either the elevator angle or the angle of attack. In-flight values can be obtained directly from sensors, if available. The selection of one or the other formulation depends on the availability and accuracy of on-board measurement equipment. On the current platform both variables are measured; so both alternatives are feasible.

The reference elevator angle can be calculated analytically from the aircraft equations of motion, however this generally requires iterative solving, which is not amenable for online applications. More efficient alternatives include pre-computing solutions for different flight conditions and storing these in lookup tables for on-board use, or deriving simple empirical expressions to compute approximate solutions more conveniently. The latter approach is used here.

The reference angle of attack can be calculated from the lift curve in a clean configuration,

$\alpha_{\mathrm{ref}}=\frac{C_{\mathrm{L}}-C_{\mathrm{L} 0}}{C_{\mathrm{L} \alpha}}$,

where $C_{\mathrm{L} 0}$ and $C_{\mathrm{L} \alpha}$ depend on Mach number and are known for a particular aircraft, and $C_{\mathrm{L}}$ can be computed in flight from Eq. 2. The above equation is valid only if the angle of attack is small, which is typically the case for conventional, fixed-wing UAS.

As before, icing is detected from differences between inflight measured and ideal values for the considered variables. For this approach, however, no data were available to define icing severities for the test platform. Instead, initial icing intensity levels were defined with the help of literature values. Data from the NASA icing flight testing conducted on the de Havilland Twin Otter aircraft [26, 28] were used to create a basic icing effects model. This model was then interfaced with an existing 6 degree-of-freedom flight dynamics model of the Jetstream [15] and the resulting changes in elevator trim and angle of attack due to icing were computed. The previously obtained information on icing-related drag changes was incorporated in the icing model to enhance the accuracy with respect to the actual platform. Evidently using data for a different aircraft entails limitations; however, the obtained results are a first approximation and can be improved when data for the actual platform become available. Thresholds representing different icing severities were derived under the same assumption made previously, i.e. that icing severity linearly relates to changes in the pertinent aerodynamic coefficients.

The computed results are presented in Table 2. Values are shown for different velocities, as they are dependent on 
Table 2 Icing severity levels for the Jetstream 31, in terms of changes in elevator trim angle $\Delta \delta_{E 0}$ and trim angle of attack $\Delta \alpha_{0}$, based on a combination of literature data and empirical data

\begin{tabular}{|c|c|c|c|c|c|c|c|c|}
\hline \multirow[b]{2}{*}{ EAS (kts) } & \multicolumn{4}{|c|}{$\Delta \delta_{E 0}\left({ }^{\circ}\right)$ for icing severity } & \multicolumn{4}{|c|}{$\Delta \alpha_{0}\left({ }^{\circ}\right)$ for icing severity } \\
\hline & Trace & Light & Moderate & Severe & Trace & Light & Moderate & Severe \\
\hline 140 & -0.05 & -0.10 & -0.17 & -0.23 & 0.17 & 0.33 & 0.52 & 0.69 \\
\hline 150 & -0.03 & -0.06 & -0.10 & -0.13 & 0.14 & 0.28 & 0.45 & 0.59 \\
\hline 160 & -0.01 & -0.02 & -0.04 & -0.06 & 0.12 & 0.24 & 0.38 & 0.51 \\
\hline 175 & 0.01 & 0.02 & 0.02 & - & 0.10 & 0.19 & 0.31 & - \\
\hline 200 & 0.02 & 0.07 & 0.10 & - & 0.07 & 0.14 & 0.22 & - \\
\hline
\end{tabular}

airspeed. Values for severe icing at high airspeeds are not reported in either case, as these represent conditions outside the flight envelope of the test platform. As expected, the change in angle of attack caused by the same amount of icing is smaller at higher velocities, where a smaller angle difference is needed for the same lift increase. The elevator angle, however, displays a less clear trend. At low velocities, as expected, the trim angle required to compensate for icing is negative and the trim angle change required to compensate for the same amount of icing decreases with increasing velocities. However the change in elevator trim angle reverses at higher velocities. This is most likely because due to the high position of the tail on the Jetstream, the tail drag generates a significant counter moment. Hence at high velocities and high icing intensities, where tail drag increases considerably, the elevator angle must increase even to generate less negative lift and less drag.

The simulation run to determine the thresholds already indicates possible limitations of this approach. While there is indeed a clear effect of icing on the required trim values, this effect may be too small to be used effectively for detection purposes. In particular, the changes in elevator trim angle are in the same approximate range as sensor inaccuracy. The elevator angle-based method has the additional drawback of the sign reversal occurring at high velocities. This phenomenon may be more pronounced for the specific platform used here; however, it should be considered. Angle of attack changes are more significant, but still small enough to require high accuracy measurements that may not be available. Hence, this method is considered less suited for application in practice. However, it should be considered that the current threshold values are based on simulation and on empirical data largely collected on a different aircraft, therefore only flight testing can ultimately determine the feasibility.

This method, like the previous, is only applicable in steady level flight, and this requirement must be fulfilled with a high accuracy. Furthermore, the location of the aircraft centre of gravity during flight must be known and sudden shifts in it must be avoided, as these will affect the trim values. Additional limitations include the necessity of measuring the elevator deflection or angle of attack with a sufficient degree of accuracy. In particular, both the reference and the iced values must be determined to within confidence intervals smaller than the relevant changes in trim, which are very small. It is possible that changes in trim will be smaller than the measurement uncertainties introduced by sensors or calculation, and this issue must be investigated thoroughly to ensure that this method is applicable.

\section{Decision-making system}

This section describes the basic architecture and initial implementation of the IRDMS. The suggested system is intended to perform the main reasoning tasks typically performed by a pilot on manned aircraft. It identifies potential icing conditions from atmospheric data given by on-board sensors, and then uses ice detection sensor data and aircraft performance data to determine whether ice is in fact forming, how severe it is, and what its effects on the aircraft are. Through fusion of the aforementioned data, the system evaluates the current situation and suggests appropriate responses to it, considering also available meteorological forecast data for the intended route.

The decision-making system is based on a belief-desireintention (BDI) agent architecture [27], designed to emulate rational human reasoning in dynamic domains. Agents possess a degree of autonomy and can perceive their environment and react to changes in it. A BDI agent, specifically, has a set of goals (desires), and attempts to achieve these by selecting appropriate actions to execute from those available to it, considering the information it has about the world (beliefs). Actions chosen for execution represent the agent's intentions. The agent should be able to commit to its plans, but also to reconsider them if necessary, e.g. in the light of new information. In the icing context, the IRDMS has the goal of maintaining safe flight at all times; it does this by monitoring the icing situation, and therefore holds a set of beliefs on the environment that come to it through sensor data; its intentions are the responses it suggests in reaction to changes in the environment, and may change when the environment changes.

The high-level structure of the IRDMS is shown in Fig. 1. The following sections outline the icing detection 


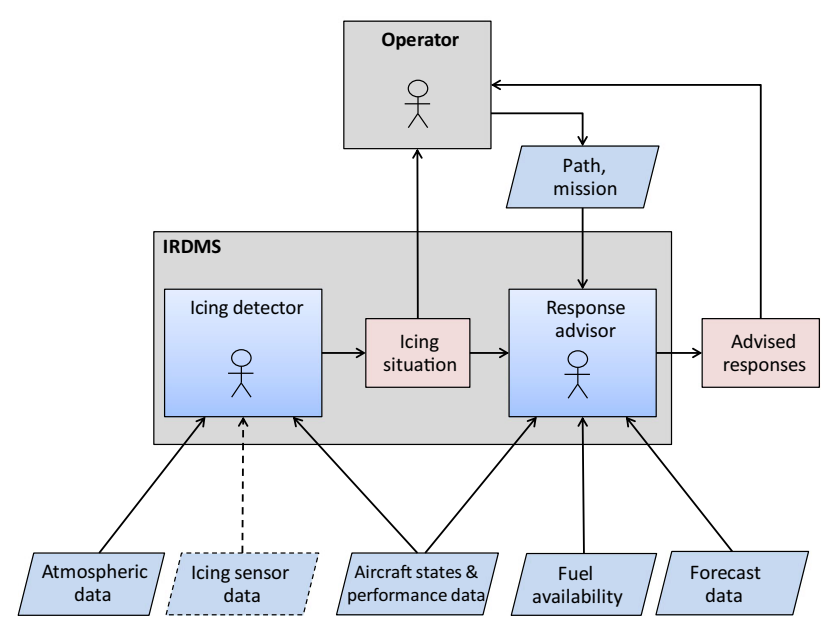

Fig. 1 Structure of the proposed icing-related decision-making system. The icing detector uses sensor data to determine the current icing situation and communicates it to the response advisor and the operator. The response advisor considers the icing situation in conjunction with forecast data and aircraft performance data to determine whether a response is required. If this is the case, it communicates the advised response to the operator

and decision-making processes. Further detail is given in [6]. The system was implemented using a commercially available agent-oriented software framework, known as JACK, developed by AOS [3].

\subsection{Detection and quantification of icing conditions}

To detect and quantitatively assess icing conditions, the IRDMS uses atmospheric data, viz. outside air temperature (OAT) and relative humidity, aircraft performance data, and information from an icing sensor, if available. Whilst civil aircraft are not always equipped with icing sensors, as the pilots can observe ice formation on the windshield, engine nacelles, etc., UAS intended to fly in icing conditions are considered more likely to possess such a sensor given the absence of a pilot. The basic structure of the icing detection component is illustrated in Fig. 2.

To begin with, the atmospheric data enable the IRDMS to determine whether the aircraft is in potential icing conditions. Changes in performance or aircraft behaviour alone may be due to any of a number of causes, thus it is essential to first determine whether icing is possible. For this to be the case, the temperature must be below freezing point and the relative air humidity sufficiently high.

Next, the IRDMS must determine whether ice is actually forming and how severe any possible build-up is. If the aircraft is in potential icing conditions, the IRDMS will assume that any deterioration in performance or change in trim settings in the absence of known failures, is a consequence of icing. It will determine a separate icing severity value by means of each of the two methods outlined in
Sect. 2. Comparing the icing severity suggested by each method provides a means for corroborating the conclusion drawn and identifying possible problems. Additionally, readings from an icing sensor are included to provide a degree of information redundancy. Evidently even in potential icing conditions, there may be other issues affecting the aircraft's performance, stability and control; so it is essential to consider more than one source.

Thus the IRDMS computes separate icing severity levels from each of the available sources. These values are then compared and evaluated in combination. At present the data fusion process is fairly simple and based on the extent of agreement of redundant information sources and the likelihood of particular failures occurring or measurements being erroneous or inaccurate. At the lowest level, responses are also based on a worst case scenario, so that in case of uncertainty it is always ensured that the aircraft remains safe. Essentially, the process imitates the reasoning process of a human. If the available information sources all agree, a conclusion can easily be drawn. In general, the agreement should be sufficient for the overall icing severity to be determined with acceptable confidence. It is, however, possible for there to be discrepancies between the different sources. In this case there is more than one possible interpretation, and the system will look for and attempt one of these.

In the case of minor discrepancies, the worst case is assumed, in the interest of safety, but typically no failure is suspected. Different approaches have different accuracies and use different data, and will often yield slightly different results, particularly considering the discrete and very approximate icing severity thresholds defined. A situation of slight discrepancy is in fact the most likely to occur.

If more significant disagreements occur, the IRDMS attempts to resolve these by considering possible failures and determining the most likely cause of these. Failures may for instance be a malfunctioning sensor, or structural damage to the aircraft. Nonetheless, it may be possible to resolve this type of situation, particularly if only one source of information is in disagreement with the rest. If possible, the IRDMS will draw a conclusion based on the remaining measurements, again, erring on the side of caution, however if the disagreement is significant, it will be signalled to the operator. Similarly, if the disagreement is such that no conclusion can be drawn with sufficient confidence, and several different sources are all giving different information, a warning is issued to the operator. Typically, in this case the problem is no longer a responsibility of the IRDMS and becomes a general issue that must for instance be addressed via fault-detection methods.

The IRDMS has a number of possible interpretations and plans at its disposal which it can fit to the perceived situation. If none of the interpretations fit, it signals the 
Fig. 2 Ice detection component or the IRDMS. This component collects data on the atmospheric conditions, the weather, and the aircraft state and performance, and uses this to establish whether ice is forming and how significant its effects on the vehicle are. Information from an icing sensor is also used, if available

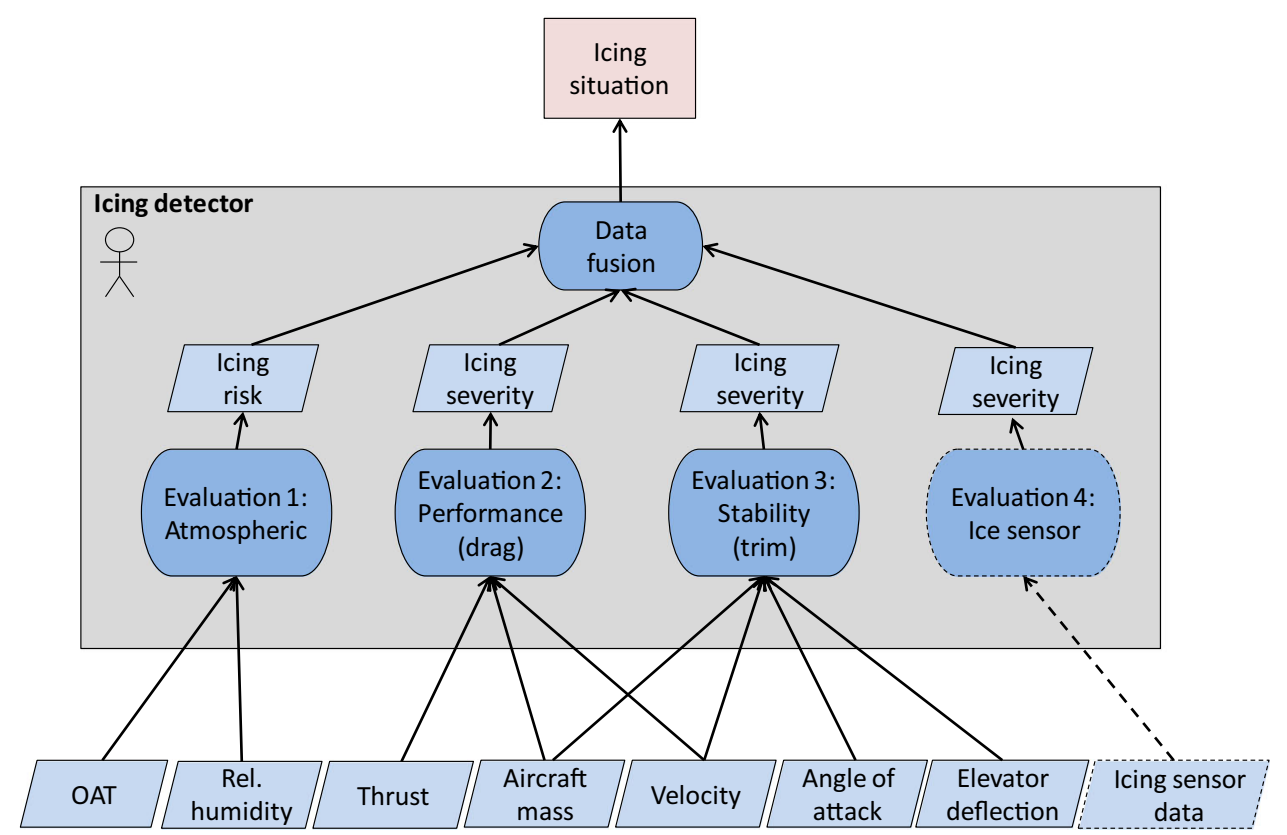

situation to the operator as a warning. While not all cases can be considered, the plans are defined as generically as possible to ensure that they each cover several different but similar situations and that the system is not excessively limited. If for instance the aircraft is in potential icing conditions and no performance deterioration is detected, only the activation of the anti-icing system is advised. If the aircraft is in icing conditions and discrepant information is given by different sources, the IRDMS either assumes the worst case, or the most likely, depending on how significant the discrepancy is and whether there is any partial agreement between different sources. If the aircraft is clearly not in icing conditions and performance changes are detected, these are assumed to be due to other causes. Depending on the magnitude of these changes, they may be signalled to the operator, who can then consider further measures.

\subsection{Responses}

The response component of the IRDMS is tasked with selecting an appropriate response to the perceived icing situation determined by the ice detection component described in Sect. 3.1. The responses advised depend on the detected icing situation, as well as on additional information, such as the weather forecast for the current location and for the intended path, meteorological reports from other vehicles, fuel availability and aircraft performance. While there is an underlying guideline to cover the most common occurrences and ensure a basic degree of safety, the decision-making system is designed to be flexible and essentially modular, so that it can be extended and refined at leisure to cover additional cases, e.g. based on experience gained in the testing and design process. The response advisor component has a set of plans at its disposals, which it considers in turn when evaluating a specific situation. Figure 3 illustrates the possible decisionflow after determination of the icing situation, and in the specific case of severe icing. In each case, different questions are considered by the system in turn, and based on this an appropriate course of action is selected from the ones available.

If the IRDMS has determined that icing is possible, its immediate response is to advise activating the anti-icing system. This is preventive and counteracts the formation of ice in favourable conditions. Therefore, in compliance with icing regulations for manned aircraft, it should be activated as soon as an aircraft is in conditions where ice may form. Further measures depend on whether icing is detected by any available detection component. As soon as ice appears to be forming, the de-icing system is activated. Managing the de-icing system requires knowledge on the amount of ice on the airframe, as the system must be activated at intervals, only when there is sufficient ice for it to be effective. A pilot would tackle this task by looking at the wing, but on a UAS different means must be found to gauge the amount of ice on the airframe. While the detailed implementation of the system has not yet been designed, it is envisaged that the de-icing system will be controlled according to the estimated icing level and using information from sensors.

Next to the activation and management of the IPS, additional measures may be required, particularly if severe icing has been detected or forecast for a future stretch of 
(a)

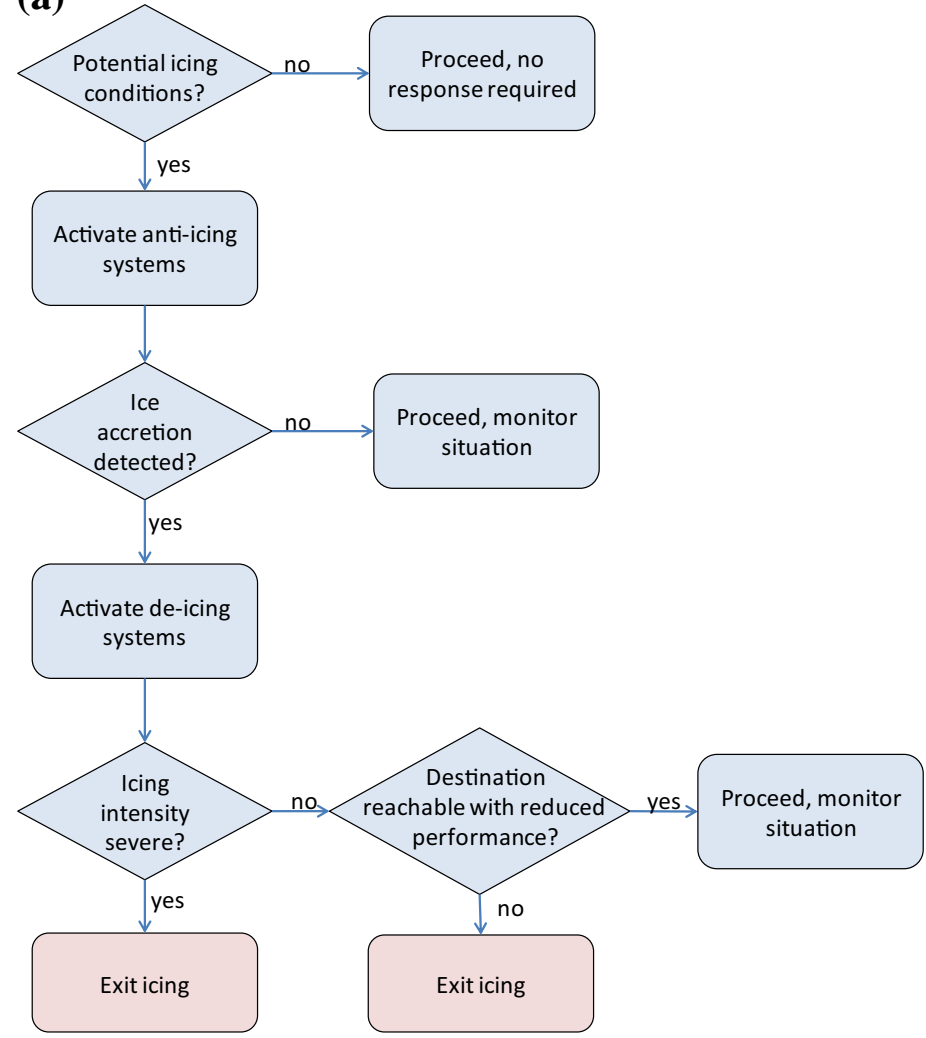

(b)

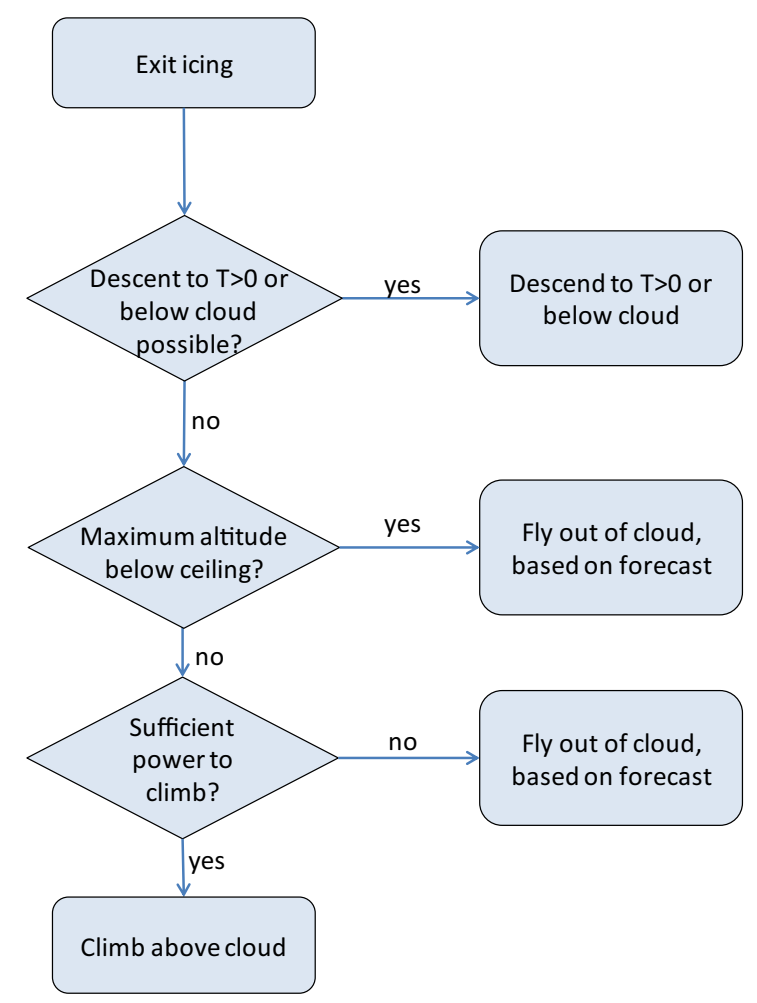

Fig. 3 Basic decision-flow for two components of the decision-making process

the intended flight path, or if the aircraft has been exposed to icing for extended time periods. Action may also be required as a consequence of previous incidents, so the IRDMS must continually monitor the state of the aircraft.

In response to less than severe icing, advice to activate the IPS may be followed by a fuel availability check, assuming the perceived icing situation is protracted, and if applicable also considering updated forecast information. The aircraft may proceed as long its performance is within acceptable bounds and does not take it close to flight envelope limits, and there is enough fuel for the destination to be reached. If this is no longer the case, a new path is searched for. Thanks to fuel reserves, the critical issue is more likely to be excessive performance deterioration, leading to a limited flying capability or stability and control problems, rather than a long-term effect.

If severe icing has been detected or if the increased fuel consumption due to icing would not allow for the intended destination to be reached, exiting icing conditions is advised. Depending on the case, this may involve more extensive path planning, but typically consists of a basic immediate avoidance manoeuvre, where the aircraft resorts to the most convenient and rapid route to safety that is feasible. This could be to climb out of cloud or descend below cloud and/or to higher temperatures. In considering which action to take, the IRDMS takes into account the fuel availability, current flight conditions, aircraft state and performance, and atmospheric/meteorological information.

A flight path change is also advised if severe icing is expected farther along the intended path. This is based on meteorological information, which this type of UAS is assumed to have access to, either from standard sources (e.g. METAR), or through communication with other aircraft or UAS (PIREPS or similar). Given that there is a degree of uncertainty associated with forecast information, only severe icing forecasts are considered in order to avoid unnecessary disruptions from the intended mission. If an icing forecast is available, the system attempts to compute a new path that avoids regions of severe icing. If at any point it appears that only a single airfield can be reached, then landing is suggested, but again this represents an extreme case.

In general, the aircraft is allowed to proceed on the intended path as long as it does not encounter severe icing, its performance deterioration is not excessive, and at least two airfields can be reached according to the current situation and the forecast along the planned path. Where possible no major re-planning takes place, and the aircraft is maintained on its path or returned to it as soon as the situation permits. This solution ensures an adequate safety 
level without excessively affecting the chances of mission completion.

Finally, it is possible for the IRDMS to be unable to draw conclusions from the available data, or to detect aircraft behaviour changes that seem unrelated to icing. In such cases, the IRDMS will notify the operator, who must then decide on an opportune course of action.

\section{Simulation and flight testing}

\subsection{Simulation}

Initial tests were conducted in a simulation environment to verify the software implementation, demonstrate the basic framework and functionalities of the IRMDS, and evaluate whether the suggested approach is feasible. The simulation framework comprised three parts, viz. the previously mentioned MATLAB/Simulink model of the Jetstream 31 flight dynamics, the IRDMS, implemented in JACK, and open-source simulator FlightGear, which was used for visualisation purposes and as an interface channel between the two other components [22, 24].

To allow for more realistic dynamic simulation, the static icing effects model introduced in Sect. 2.2 in the context of defining icing severity thresholds, was extended to account for time effects. Icing was modelled as a gradual deterioration of the aerodynamics in time, rather than a fixed, instantaneous reduction as before. The time-dependent rate of ice accretion was expressed as a rate of change of a number of relevant aerodynamic coefficients (mainly $C_{\mathrm{D} 0}, C_{\mathrm{L} \alpha, \mathrm{w}}, C_{\mathrm{L}, \alpha, \mathrm{t}}, C_{\mathrm{m} \delta_{\mathrm{E}}}$ ). With the help of experience-based suggestions from pilots, assumptions were made on how much time it would take to reach the nominal threshold values defined previously in the static model. Given that the model is based on the same assumptions as one of the detection approaches, it does not allow for the threshold values to be validated; however, it provides a useful framework for simulation and verification purposes. These tests were aimed at showing that the main processes work correctly and the system can satisfactorily respond to different occurrences.

Initially, static tests were run, where the system was placed in a specific, unchanging situation and its interpretation of the situation and immediate response were observed. This was to test whether the system can correctly interpret different combinations of information and whether its immediate response is reasonable. For this, the aircraft was trimmed in different steady level flight conditions with an autopilot, and ice was allowed to build up at different rates, representing different intensities. As the ice builds up, the dynamics of the aircraft are affected, and the response of the IRDMS can be observed. Next to interpreting the information given by each ice detection approach, the tasks of the IRDMS include merging the information given by different sources, identifying and approximately quantifying icing from this, and suggesting adequate responses to the identified conditions.

A number of more complex simulations were also conducted to verify and test the decision-making component of the IRDMS more extensively. For this, different scenarios were considered, chosen in such a way that the main features of the system were tested and the most likely situations simulated. These scenarios involve a dynamic simulation, where the aircraft begins in conditions where no icing can occur, then flies into icing, with different situations being considered, such as: discrepant information from different sources, information changing over time, changing forecast information, occurrence of severe icing, limitations to the possible responses, suspected failures, etc.

Results show that the IRDMS responds in an informed and safe way, and complies with the guidelines defined initially. For the considered cases, the system can identify icing effectively from the available data and suggest appropriate responses. The system advises activating and de-activating the IPS as required, ensures that aircraft performance is sufficient for continued safe flight, suggests appropriate options to fly out of icing conditions if severe icing occurs, and advises path re-planning if severe icing is forecast for the intended route. Discrepant data provided to the system was interpreted reasonably, with responses ensuring continued safety of the aircraft.

The simulation tests show that the software is correctly implemented. An evaluation and validation of the proposed detection methods, as well as testing whether the implemented IRDMS can function on board a real aircraft, requires a flight test programme. Initial tests were conducted and are outlined subsequently.

\subsection{Flight testing}

Initial flight testing was conducted on the Cranfield University Jetstream 31, with the aim of assessing the effectiveness and applicability of the suggested icing detection approaches in a real-life context, and evaluating the functionality of the IRDMS, when implemented on board an aircraft. The IRDMS was run on a laptop that was plugged into the Jetstream's on-board computer, and continually received aircraft sensor values in the form of streamed UDP data.

The Jetstream was flown in three different trimmed steady flight conditions, at velocities (indicated airspeeds) of $V_{0}=120,150,200 \mathrm{kts}$, first in the absence of icing, to obtain baseline values, and then in icing conditions. Ice was allowed to form naturally, by flying the aircraft into 
cloud in conditions favourable for ice accretion. To allow for performance degradation to be evaluated more effectively, the de-icing system on the wings and tailplane was not activated; however, anti-icing protection on the propellers and engine inlets was always activated to comply with the aircraft flight manual. This does not have an effect on the results. Values for the ice-affected aircraft began to be recorded once a visible layer was remarked on the leading edges of the lifting surfaces. After completion of each set of measurements, the de-icing boots were activated, and the aircraft returned to a clean condition prior to the successive testing phase.

The icing severity level was judged by the pilots on board based on their experience, in combination with weather forecast analysis. This implies that the judgement is to some extent subjective; however, at present in aviation icing severity is in fact judged by the pilot and there is no more systematic alternative. Given that flight in severe icing is not permitted, such conditions could not be tested. Additionally, weather conditions on the flight days only allowed for trace and light icing to be tested. The drawback of flight testing in the icing context is that unless icing is simulated, the type and extent of icing that occurs depends on the meteorological conditions on the flight day, and is difficult to predict accurately. An evaluation of the trimbased detection method was only possible to a limited extent, as external disturbances influenced the final results; therefore, it has not been discussed here.

Figure 4 shows the drag coefficients computed from inflight measurements, both in a clean configuration in the absence of icing, and in trace and light icing conditions, plotted against the square of the corresponding lift coefficient, and against aircraft velocity. It can be seen that icing appears to have a visible effect on the drag coefficient, suggesting that in principle the suggested method can provide a characterisation of in-flight icing. However a quantitative evaluation was found to be challenging as there was a high degree of noise in the measurements required to compute the drag coefficient in flight. Particularly in trace icing the measured changes in drag were in the same order of magnitude as the error associated with the computed clean $C_{\mathrm{D}}$ values, thus making it difficult to effectively distinguish the effects of icing from the effects of noise, or to determine to what degree changes in the drag were caused by one effect or the other. This suggests that more accurate or filtered measurements are required for this method to be applicable. Even then, it is possible that this approach will not be adequate to identify trace and light icing with accuracy, thus further testing should evaluate this.

Due to the closeness between drag changes and error bounds, it was also not possible to obtain more accurate thresholds for different icing severities and evaluate the initially chosen values effectively, particularly given that only trace and light icing were encountered in flight and no experimental data were, therefore, available for moderate and severe icing.

In spite of the limitations of the current flight testing, results suggest that the proposed drag-based method is promising as a means of ice detection, as changes in drag coefficient were noticeable even in light icing. The approach seems more suitable for the determination of moderate or heavy icing; however, the use of more accurate sensors or of filters may also allow for trace and light icing to be recognised more effectively. Finally, it must be noted that further evaluations would be needed to assess (a)

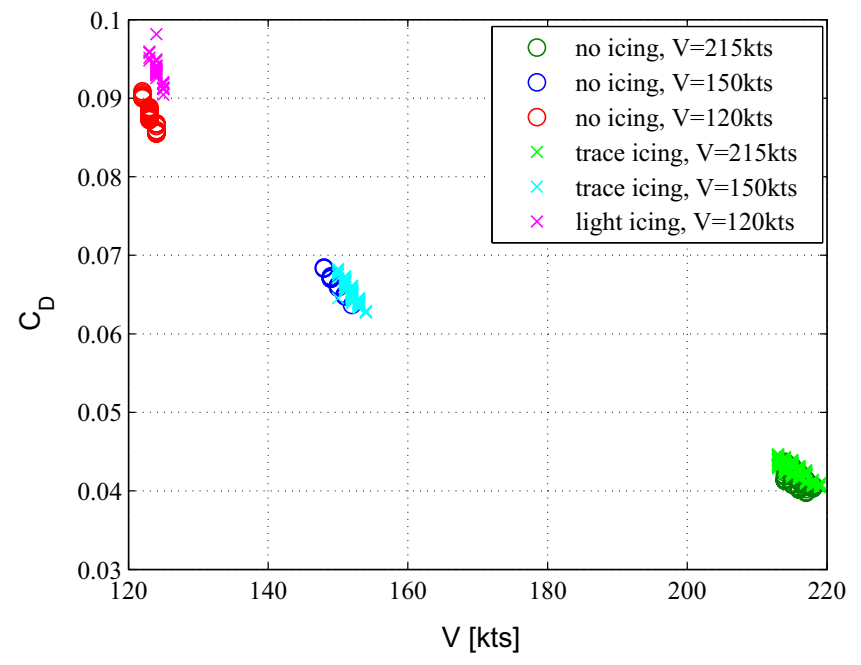

(b)

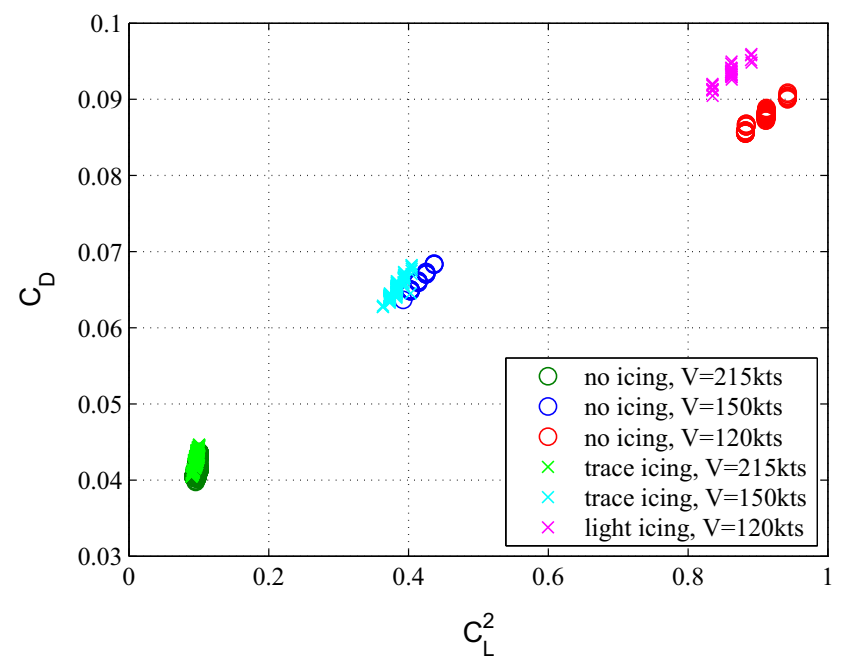

Fig. 4 Flight test results evaluating the effect of icing on the drag coefficient 
the suitability of the approach for smaller, lighter vehicles. These would be more affected by icing, but the changes caused would also likely have a smaller order of magnitude. An evaluation of the trim-based detection method was only possible to a very limited extent due to external disturbances during the tests; therefore, it has not been discussed here.

On a software level, it was shown that the IRDMS can communicate with the aircraft's on-board computer and evaluate sensor data in real time without problems. The system was readily integrated with the aircraft's sensor systems and flight management system.

\section{Conclusions}

An icing-related decision-making system (IRDMS) was designed, with the aim of enabling UAS to operate safely in icing conditions that their IPS can handle. The IRDMS uses atmospheric measurements, ice sensor data, and aircraft dynamics and performance data to detect and quantify icing in flight. Having established the icing situation, it determines an appropriate response to it if necessary, so as to maintain the vehicle safe without excessively affecting its operations. Responses include the activation of protection systems, diversion manoeuvres and path re-planning. Meteorological forecast data is also considered so that pre-emptive measures can be taken when severe icing is expected. Suggested responses and relevant information are communicated to the operator on the ground, to facilitate his decision-making process, which is hindered by his lack of visual and sensory cues.

To support the IRDMS in recognising icing, two icing characterisation methods for steady level flight were proposed, which quantify icing from changes in drag coefficient and trim settings, respectively. Thresholds defining icing severities were derived from experimental data for the test platform and literature data. Initial flight testing suggests that the drag-based method is viable, but would require more accurate measurements or filtering, particularly to allow for trace and light icing to be identified. While trends were clearly visible, a quantitative evaluation of the icing severity threshold values was difficult due to the similar magnitude of the icing-related drag changes and the error associated with the in-flight measurements. Prior to further flight tests, methods to increase the accuracy of the measurements should be considered.

The IRDMS was tested in a simulation environment to demonstrate its basic functioning and verify the software implementation. For this, a basic icing accretion model was developed and interfaced with a flight dynamics model of the test platform. Results show that the IRDMS recognises icing effectively and suggests appropriate responses to it, which keep the vehicle in safe conditions and comply with existing guidelines for manned aircraft. A real-time implementation of the proposed system would enhance UAS capability for operating in adverse weather conditions, thus increasing their potential range of application. The current prototype is considered a useful starting point, that can be extended to cover more situations and incorporate more advanced and efficient icing detection methods and reasoning processes.

Further work is planned, starting from more extensive testing to evaluate the icing characterisation techniques and to validate and refine the current icing severity thresholds. Wind tunnel tests in particular, will also allow for severe icing to be investigated. The icing characterisation component must also be extended to cover different flight conditions, including manoeuvring flight. Some form of flight envelope protection will additionally be considered, as even when performance deterioration is not excessive per se, it is essential not to cross the boundaries of the reduced flight envelope, e.g. through manoeuvring. While re-estimating the flight envelope online is challenging, particularly on small UAS, possibilities to provide some information on the reduced flight envelope and avoid critical conditions will be investigated. Finally, more extensive testing will be conducted to investigate the reliability and robustness of the system.

Acknowledgments The authors thank Dr Alastair Cooke for the Jetstream flight dynamics model, Gaetan Martin for his work on the simulation framework, and the Cranfield National Flying Laboratory Centre for the flight testing. This work was funded by Innovate UK and Agent Oriented Software Limited, as part of the Autonomous Systems Technology Related Airborne Evaluation \& Assessment (ASTRAEA) Programme.

Open Access This article is distributed under the terms of the Creative Commons Attribution 4.0 International License (http://crea tivecommons.org/licenses/by/4.0/), which permits unrestricted use, distribution, and reproduction in any medium, provided you give appropriate credit to the original author(s) and the source, provide a link to the Creative Commons license, and indicate if changes were made.

\section{References}

1. ASTRAEA. Website. http://www.astraea.aero/

2. Final Report on the accident on 1st June 2009 to the Airbus A330-203 registered F-GZCP operated by Air France flight AF447 Rio de Janeiro-Paris. Bureau d'Enquêtes et d'Analyses pour la sécurité de l'aviation civile. http://www.bea.aero/en/ enquetes/flight.af.447/rapport.final.en.php

3. JACK An Agent Infrastructure for Providing the Decision-Making Capability Required for Autonomous Systems. Tech. rep., AOS Group. http://www.aosgrp.com/downloads/JACK_White Paper_UKAUS.pdf 
4. Addy, H., Lee, S.: Icing encounter duration sensitivity study. In: AIAA Atmospheric and Space Environments Conf., San Antonio, TX (2009)

5. Addy, H.E., Chung, J.J.: A wind tunnel study of icing effects on a natural laminar flow airfoil. In: 38th AIAA Aerospace Sciences Meeting \& Exhibit, Reno, NV (2000)

6. Armanini, S. F.: Autonomous decision-making for UAV flight in icing conditions. Semester thesis (TU München), Cranfield University, 2012 (unpublished)

7. BAE Systems. Jetstream Series 3102 Crew Manual 08C (2007)

8. Bower, D.R.: The continuing threat to aviation safety from aircraft icing a review of recent NTSB accident and incident investigations. In: SAE 2007 Aircraft \& Engine Icing International Conference, Seville, Spain (2007)

9. Bragg, M., Basar, T., Perkins, W., Selig, M., Voulgaris, P., Melody, J.: Smart Icing systems for aircaft icing safety. In: 40th AIAA Aerospace Sciences Meeting \& Exhibit, Reno, NV (2002)

10. Bragg, M., Broeren, A., Blumenthal, L.: Iced-airfoil aerodynamics. Prog. Aerosp. Sci. 41(5), 323-362 (2005)

11. Bragg, M., Hutchinson, T., Merret, J., Oltman, R., Pokhariyal, D.: Effects of ice accretion on aircraft flight dynamics. In: 38th AIAA Aerospace Sciences Meeting \& Exhibit, Reno, NV (2000)

12. Bragg, M.B., Perkins, W.R., Sarter, N.B., Basar, T., Voulgaris, P.G., Melody, J.W., McCray, S.A., Gurbacki, H.M.: An interdisciplinary approach to inflight aircraft icing safety. In: 36th Aerospace Sciences Meeting \& Exhibit, Reno, NV (1998)

13. Broeren, A.P., Whalen, E.A., Busch, G.T., Bragg, M.B.: Aerodynamic simulation of runback ice accretion. J. Aircr. 47(3), 924-939 (2010)

14. Caliskan, F.: Neural Network Based Icing Identification and fault tolerant control of an A340 aircraft. Int. J. Mech. Ind. Mechatron. Eng. 1(4), 174-179 (2007)

15. Cooke, A.A.: Simulation model of the NFLC Jetstream 31. Tech. Rep. COA-0402, Cranfield University (2006)

16. Cristofaro, A., Johansen, T.A., Aguiar, A.P.: Icing detection and identification for unmanned aerial vehicle: multiple model adaptive estimation. In: European Control Conf., Linz, Austria (2015)

17. Di Donato, P.F.A., Yoneyama, T., Macau, E.E.N.: Airplane ice accretion detection using parameter estimation. Navigation and Control Conf., Minneapolis, MN, In AIAA Guidance (2012)

18. Dong, Y., Ai, J.: Inflight parameter identification and icing location detection of the aircraft: the time-varying case. J. Control Science and Engineering (2014)

19. Han, Y., Palacios, J.: Analytical and experimental determination of airfoil performance degradation due to ice accretion. In: AIAA Atmospheric and Space Environments Conf, New Orleans (2012)
20. Jeck, R.K.: DOT/FAA/AR-01/91 A History and Interpretation of Aircraft Icing Intensity Definitions and FAA Rules for Operating in Icing Conditions. Tech. rep., U.S. Dept. of Transportation Federal Aviation Administration (2001)

21. Lucas, C.A.: The effects of icing on the dynamic response of thick aerofoils. Aeronaut. J. 95(947), 231-240 (1991)

22. Martin, G.: Modelling, interfacing and visualisation of the Jetstream J31. Internship rep., Cranfield University (2012) (unpublished)

23. Melody, J., Basar, T., Perkins, W., Voulgaris, P.: Parameter identification for inflight detection and characterization of aircraft icing. Control Engineering Practice 8 (2000)

24. Polak, M. Identification and simulation of ice accretion and its effects on aircraft. Semester thesis (TU München), Cranfield University (2013) (unpublished)

25. Ranaudo, R., Mikkelsen, K., McKnight, R.: Performance degradation of a typical twin engine commuter type aircraft in measured natural icing conditions. Tech. Memorandum 83564, NASA (1984)

26. Ranaudo, R.J., Mikkelsen, K.L., Mckmight, R.C., Ide, R.F., Reehorst, A.L., Jordan, Jerry, L., Schinstock, W., Platz, S.J. The measurement of aircraft performance and stability and control after flight through natural icing conditions. In: AIAA/AHS/ CASI/DGLR/IES/1SA/ITEA/ SETP/SFTE 3rd Flight Testing Conf., Las Vegas, NV (1986)

27. Rao, A.S., Georgeff, M.P. Modeling rational agents within a BDIarchitecture. In: Proc. of the 2nd Int. Conf. on Principles of Knowledge Representation and Reasoning (1991)

28. Ratvasky, T., Ranaudo, R.: Icing effects on aircraft stability and control determined from flight data. Tech. Memorandum 105977, NASA (1993)

29. Schuchard, E.A., Melody, J.W., Basar, T., Perkins, W.R., Voulgaris, P. Detection and classification of aircraft icing using neural networks. In: 38th AIAA Aerospace Sciences Meeting \& Exhibit, Reno, NV (2000)

30. Sørensen, K.L., Blanke, M., Johansen, T.A.: Diagnosis of wing icing through lift and drag coefficient change detection for small unmanned aircraft. IFAC-PapersOnLine 48(21), 732-739 (2015)

31. Tousi, M.M., Khorasani, K. Robust observer-based fault diagnosis for an unmanned aerial vehicle. In: Systems Conference (SysCon), 2011 IEEE International, pp. 428-434 (2011)

32. Dept, U.S. of Transport. Federal Aviation Administration, Aeronautical Information Manual (2014)

33. Whalen, E., Melody, J., Bragg, M., Basar, T. Aircraft characterisation in icing using flight test data. In: Proc. of the 42 nd AIAA Aerospace Sciences Meeting and Exhibit, Reno, NV (2004) 